\title{
Investigation of risk factors associated with objective parameters of the nutritional status in hospitalised patients
}

\author{
V. A. Leandro-Merhi and J. L. B. Aquino \\ School of Nutrition and Medicine, Pontifical Catholic University of Campinas (PUC-Campinas), SP-Brazil
}

The investigation of risk factors associated with nutritional status right after admission could contribute for better knowledge and identification of this situation, allowing better control of strategies for the primary prevention of malnutrition ${ }^{(1,2)}$. The objective of the present study was to investigate the incidence of malnutrition and its possible association with many parameters that assess nutritional status and to identify the associated risk factor.

The nutritional status was assessed in 235 hospitalised patients of both genders. The criteria to malnutrition was proposed based on anthropometric nutritional status parameters. The malnutrition was defined as present when the patient presented at least two anthropometric criteria below the normal range and habitual energy intake below $75 \%$ of the energy requirement $(\mathrm{HEI} / \mathrm{ER}<75 \%$ ). The anthropometric criteria were: $\mathrm{BMI}<18.5 \mathrm{~kg} / \mathrm{m}^{2}$ for adults and $<22 \mathrm{~kg} / \mathrm{m}^{2}$ for the elderly and arm circumference, triceps skinfold thickness, mid-arm muscle circumference, arm muscle area or arm fat area below the 10th percentile $(<\mathrm{P} 10)$. Gender, age, type of disease, recent weight change and dental problems were investigated as possible associated risk factors. The Chi-square and Mann-Whitney tests were used to compare the data and univariate and multiple logistic regressions were used to identify the factors associated with malnutrition. The OR and CI of $95 \%$ were calculated with the significance level set at $5 \%(P<0.05)$.

One-fifth $(20 \%)$ of the patients were malnourished on admission to the hospital and $27.5 \%$ reported recent weight loss. The malnutrition $(P<0.0001)$ was greater in patients with malignant diseases. The only variables significantly associated with malnutrition according to univariate logistic regression were recent weight loss $(P=0.0058$; OR $=2.909 ;$ IC95\% $=1.362 ; 6.212)$ and malignant disease $(P=0.0001 ; \mathrm{OR}=3.847$; IC95\% $=1.948 ; 7.597)$. When multiple regression was used in the model which included type of disease, malignant disease was shown to increase the chance of malnutrition 4 -fold $(P=0.0002 ; \mathrm{OR}=3.855 ; \mathrm{IC} 95 \%=1.914 ; 7.766)$. When disease was excluded, recent weight loss also increased malnutrition 4-fold $(P=0.0012 ; \mathrm{OR}=3.716$; IC95\% $=1.677 ; 8.236)$.

\begin{tabular}{lrrrrr}
\hline Variables & Estimate & SE & $P$ value & OR & CI (95\%) \\
\hline $1^{\circ}$ model* & & & & & \\
Intercept & -1.7707 & 0.2208 & $<0.0001$ & & \\
Malignant $\times$ benign disease & 1.3495 & 0.3573 & 0.0002 & 3.855 & $1.914,7.766$ \\
$2^{\circ}$ model** & & & & & \\
Intercept & -1.8563 & 0.2776 & $<0.0001$ & & \\
RWC: gain $\times$ not & 0.5126 & 0.4268 & 0.2298 & 1.670 & $0.723,3.854$ \\
RWC: loss $\times$ not & 1.3127 & 0.4061 & 0.0012 & 3.716 & $1.677,8.236$ \\
\hline
\end{tabular}

RWC: Recent weight change.

In conclusion, patients with a history of recent weight loss and those with malignant diseases are more susceptible to malnutrition.

This work was sponsored by Research Support Fund of the Pontifical Catholic University of Campinas (PUC-Campinas), SP-Brazil, 2010.

1. Korfali G, Gundogdu H, Aydmtug S et al. (2009) Clin Nutr 28, 533-537.

2. Pirlich M, Schutz T, Norman K et al. (2006) Clin Nutr 25, 563-572. 